\title{
Probing Interfaces Using a Combination of Scanning Transmission Electron Mi- croscopy and Density-Functional Theory
}

\author{
S. T. Pantelides, ${ }^{* * *}$ T. J. Pennycook, ${ }^{*} * *$ W. Luo, ${ }^{* * *}$ M. P. Prange, ${ }^{* * *}$ H. N. Lee, ${ }^{* *}$ M. P. Ox- \\ ley, ${ }^{* * *}$ J. Garcia-Barriocanal, ${ }^{* * *}$ F. Y. Bruno, ${ }^{* * *} \quad$ C. Leon, ${ }^{* * *}$ J. Santam aria, ${ }^{* * *}$ M. F. Chi- \\ sholm, ${ }^{* *}$ M. Varela, ${ }^{* *}$ and S. J. Pennycook**** \\ *Department of Physics and Astronomy, Vanderbilt University, Nashville, TN 37235 \\ **Materials Science and Technol ogy Division, Oak Ridge Nationa 1 Laboratory, Oak Ridge, TN \\ 37831 \\ ***Universidad Complutense de Madrid. Madrid 28040, Spain
}

Scanning transmission electron microscopy provides atomic-resolution images of crystal structures and electron-energy-loss spectra (EELS) from which one can extract structural information and the chemical identities of constituent elements. Density functional theory (DFT) amounts to a "theoretical microscope", providing equilibrium positions for atoms and calcu lated EELS for com parison with measured spectra. This talk will use several examples to highlight the power of the com bined techniques to probe the properties of complex interfaces. In addition, the talk will highlight a recent advance that holds enormous potential: diffraction theory has been combined with density functional theory for EELS near-edge structure, producing the ultimate theoretical microscope for the extraction of information from data in complex systems.

A first example of probing interf aces is the observation of colo ssal ionic conductivity in yttriastabilized-zirconia/strontium-titanate (YSZ-STO) multilayers at room temperature, a significant advance for solid-oxide fuel cells. Initial microscopy [1] demonstrated that the interfaces are atomically flat and the YSZ layer is perfectly coherent with the STO layer, despite a huge 7\% lattice m ismatch. Finite-temperature DFT simulations of strained bulk zirconia with oxygen vacancies showed that high ionic conductivity is obtained above $1500 \mathrm{~K}$, mediated by a highly disordered $\mathrm{O}$ sublattice (Fig. 1). Simulations in a trilayer found that the sam e high degree of disorder o ccurs near room temperature, accounting for the observations [2]. Subsequent experimental and theoretical O K EELS of the multilayer confirmed the high level of disorder of the O sublattice in YSZ [3] (Fig. 2).

A second example is the use of microscopy and theory to probe how interfaces cope with the need to terminate ferroelectric polarizations (Fig. 3). In one case, the data show evidence for ionic screening, which has been predicted by theory but never observed. For a ferroelectric film on an insulating substrate, on the other hand, microscopy and theory make the case that compensation is mediated by an interfacial charge generated by oxygen vacancies.

A third example is the detection of a depression in the saturation magnetization in $\mathrm{La}_{0.67} \mathrm{Ca}_{0.33} \mathrm{MnO}_{3}$ $\mathrm{YBa}_{2} \mathrm{Cu}_{3} \mathrm{O}_{7}$ interfaces on the LCMO side. Conflicting interpretations had been proposed. DFT calculations of position-dependent magnetic couplings between interlayer and intralayer Mn atoms demonstrated clearly the presence of a magnetic dead layer.

Finally, a brief note on the recent development of the theory and computer codes to combine diffraction theory and solid-state effect s in EELS calculations: Results dem onstrate that the spectra are in- 
deed a function of probe position, as observed (F ig. 4). We also find evidence of nonlocality: spectral features are not necessarily dominated by contributions from the nearest atomic columns [6].

[1] J. Garcia-Barriocanal et al., Science 321676 (2008).

[2] T. J. Pennycook et al., Phys. Rev. Lett. 104, 115901 (2010).

[3] T. J. Pennycook et al. Eur. Phys. J. -Appl. Phys., in press.

[4] M. F. Chisholm et al. Phys. Rev. Lett. 105, 197602 (2010).

[5] W. Luo et al. Phys. Rev. Lett. 101, 247204 (2008).

[6] Research at Vanderbilt Univ ersity was spon sored by DOE grant DE-FG02-09ER46554 and the McMinn Endowment. Research at Oak Ridge National Laborator y was sponsored by the Division of Materials Sciences and Engineering, Basi c Energy Sciences, of the U. S. Departm ent of Energy. Computations were performed at the National Energy Research Scientific Computing Center at Lawrence Berkeley National Laboratory.
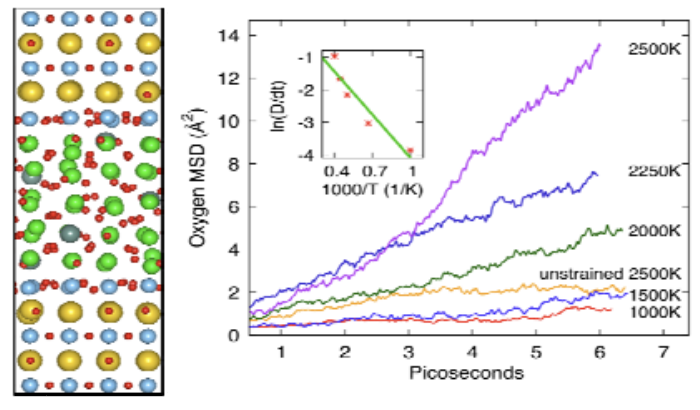

FIG. 1. Left: Structure of the YSZ/STO trilayer at $360 \mathrm{~K}$. Right: Mean squ ared displacements of $\mathrm{O}$ ions in unstrained and strained zirconia $\lceil 3\rceil$.
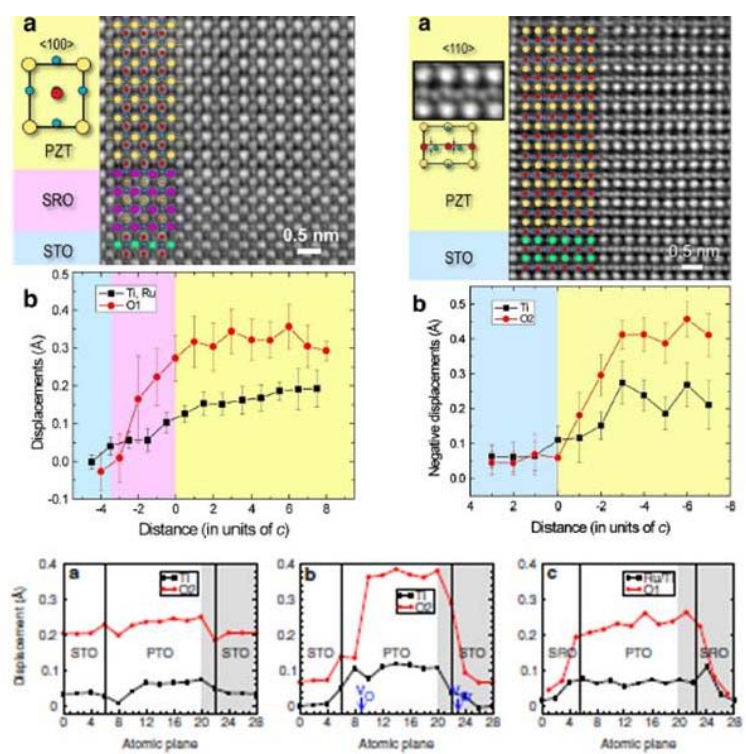

FIG. 3. Top: Phase-contrast im ages of the $\mathrm{PZT} / \mathrm{SRO} / \mathrm{STO}$ and PZT/STO st ructures and measured atomic displacements. Bottom: calculated a tomic displacements in STO/PTO/STO structures.
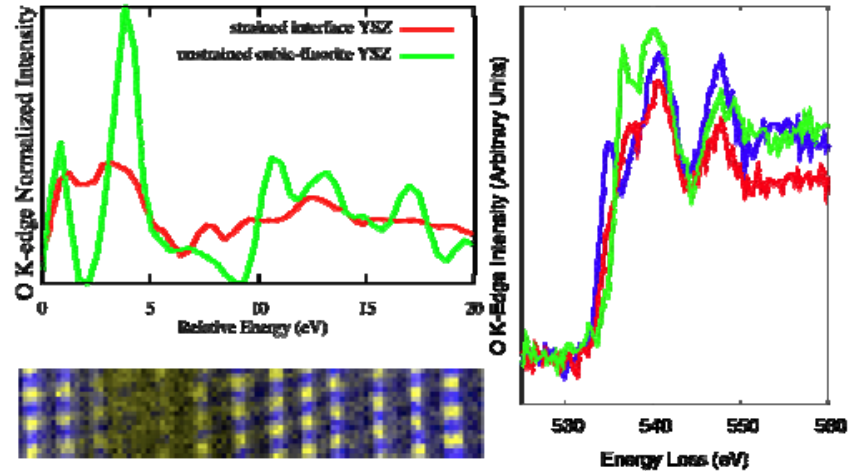

FIG. 2. (A) Calculated O K-edge averaged ov er sites in the YSZ layer compared with that of unstrained bulk. (B) Measured O K-edge in ST O (blue), strained YSZ (red) and $\mathrm{u}$ nstrained YSZ (green). (C) O (yellow) and Ti (blue) EELS m ap showing $\mathrm{O}$ disorder in strained YSZ layer.

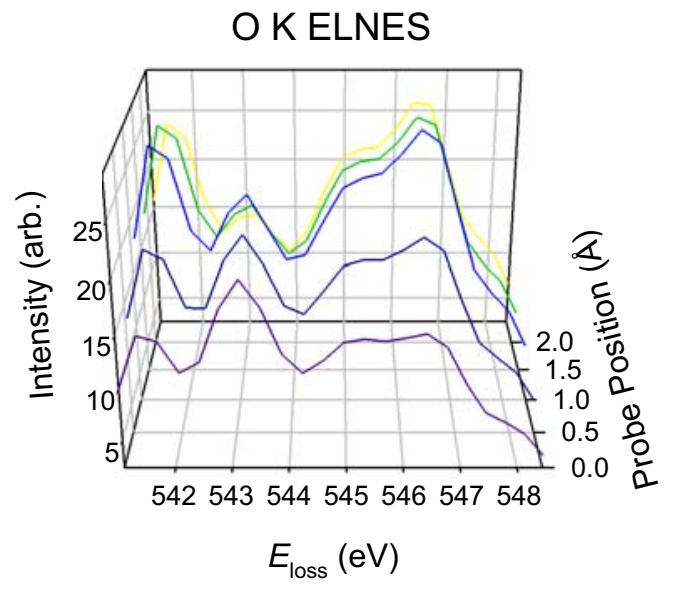

FIG. 4. O K-edge a s a function of probe position along a line extending from a $\mathrm{La} / \mathrm{O}$ colum $\mathrm{n}$ (origin) to the midpoint between $\mathrm{La} / \mathrm{O}$ columns. 